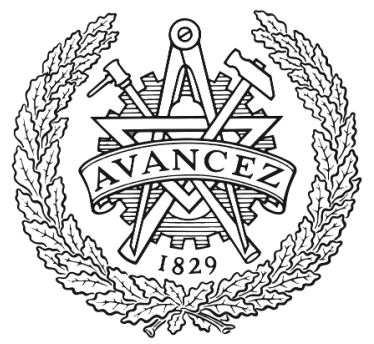

CHALMERS

UNIVERSITY OF TECHNOLOGY

\title{
Stationary wavelet processing and data imputing in myoelectric pattern recognition on a low-cost embedded system
}

Downloaded from: https://research.chalmers.se, 2023-04-26 15:19 UTC

Citation for the original published paper (version of record):

Naber, A., Mastinu, E., Ortiz Catalan, M. (2019). Stationary wavelet processing and data imputing in myoelectric pattern recognition on a low-cost embedded system. IEEE Transactions on Medical Robotics and Bionics, 1(4): 256-266. http://dx.doi.org/10.1109/TMRB.2019.2949853

N.B. When citing this work, cite the original published paper. 


\title{
Stationary wavelet processing and data imputing in myoelectric pattern recognition on a low-cost embedded system
}

\author{
Autumn Naber, Student Member, IEEE, Enzo Mastinu Student Member, IEEE, and \\ Max Ortiz-Catalan Member, IEEE
}

\begin{abstract}
Pattern recognition-based decoding of surface electromyography allows for intuitive and flexible control of prostheses but comes at the cost of sensitivity to in-band noise and sensor faults. System robustness can be improved with waveletbased signal processing and data imputing, but no attempt has been made to implement such algorithms on real-time, portable systems. The aim of this work was to investigate the feasibility of low-latency, wavelet-based processing and data imputing on an embedded device capable of controlling upper-arm prostheses. Nine able-bodied subjects performed Motion Tests while inducing transient disturbances. Additional investigation was performed on pre-recorded Motion Tests from 15 able-bodied subjects with simulated disturbances. Results from real-time tests were inconclusive, likely due to the low number of disturbance episodes, but simulated tests showed significant improvements in most metrics for both algorithms. However, both algorithms also showed reduced responsiveness during disturbance episodes. These results suggest wavelet-based processing and data imputing can be implemented in portable, real-time systems to potentially improve robustness to signal distortion in prosthetic devices with the caveat of reduced responsiveness for the typically short duration of signal disturbances. The trade-off between large-scale signal corruption robustness and system responsiveness warrants further studies in daily life activities.
\end{abstract}

Index Terms-Wavelet transforms, electromyography, signal denoising, prosthetics, data imputing.

\section{INTRODUCTION}

$\mathbf{E}$ LECTROMYOGRAPHY (EMG) signals from vestigial muscles are the most common control source for powered prostheses due to their direct correlation to motor intention and ease of non-invasive detection [1]. There is a significant discrepancy between the current mechatronic prosthetic technology and the fidelity of the signal acquisition and control systems. This results in limited controllability and frequent frustration from users [2], [3]. A study performed in 2007 showed $39 \%$ of upper-limb amputees with direct control myoelectric prostheses did not use them regularly due to issues stemming primarily from low controllability and functionality [4], though the relationship between lost limb functionality and user requirements is complex and changes over time [5]. The addition of proportional speed control and the adoption of functional hand grips by many manufacturers has not reduced

This work was supported by the Promobilia foundation, VINNOVA, the Swedish Research Council (Vetenskapsrådet), and the European Commission FLAG-ERA GRAFIN project. (Corresponding author: M. Ortiz-Catalan)

The authors are with the Department of Electrical Engineering, Chalmers University of Technology, Gothenburg, Sweden (e-mail: naber@chalmers.se; enzo@chalmers.se; maxo@chalmers.se) artificial limb rejection rates [4], [6], [7]. This suggests a more intuitive and functional control mechanism is required to address patient needs.

Direct control systems commonly used in commercial prostheses work by either cycling through grips with pre-specified muscle contractions (e.g., co-contraction of both the wrist flexor and extensor muscles) or by selecting states directly through sequences of contractions (e.g., co-contraction twice in quick succession). While users can learn these cycles and sequences, such systems limit the number of grips that are practical to use in daily life and can be slow and cumbersome to use. Using myoelectric pattern recognition (MPR) to predict motor intention from multi-channel surface EMG (sEMG) can allow more functionality and more natural use of upperlimb prostheses. With an MPR-based system, the user can change the grip of a prosthesis directly by performing a physiologically equivalent contraction as if they had an intact limb. However, the increase in functionality provided by MPR systems is offset by a large reduction in robustness. This is an important consideration, as an incorrect movement of the prosthetic at any point can compromise an entire task, hindering the clinical translation of the technology [8]. Environmental noise, signal artifacts caused by electrode movement, and missing and corrupted signals due to loose electrode-skin contact have the greatest negative impact on MPR systems using sEMG [9]-[11]. Methods that reduce their impact can have a significant positive effect on the controllability, robustness, and eventual adoption of powered prostheses. Electrode-skin decoupling and movement artifacts are typically short-term transient sources of noise. Therefore, even methods that reduce the responsiveness of the prosthesis during these events are useful if they can prevent unintended movement at critical times, i.e. avoiding the slippage of a brittle object while relocating it [12].

The aforementioned noise sources have wide-band and nonstationary characteristics. This makes them difficult to remove with FIR or IIR filters without also removing useful signal components, the effects of which are demonstrated in Fig. 1. Using more electrodes can offset some of their effects in cases of transient noise or electrode lead-off events, where the issue may only affect one channel, but this also increases the system complexity and is only useful if there are enough available myoelectric sites for recording.

Wavelet-based signal analysis has been gaining significant popularity in treating complex, noisy biological signals. Sev- 

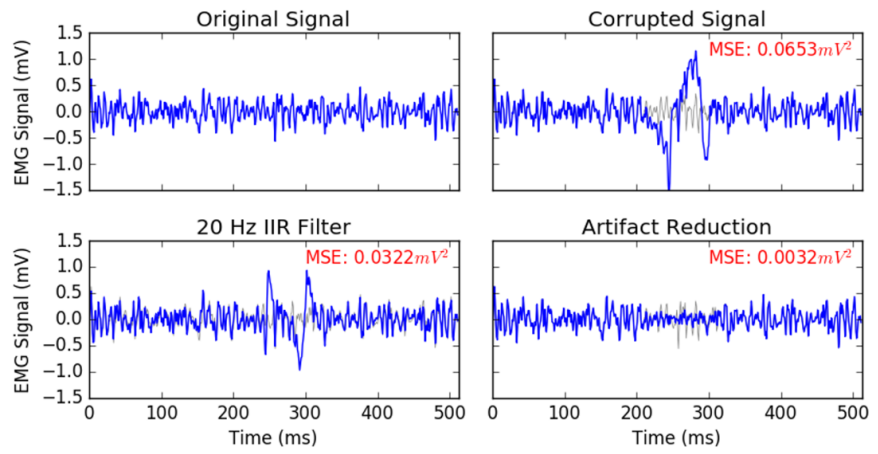

Fig. 1. Examples of clean sEMG signal (top-left) and a superimposed motion artifact (top-right) filtered with a conventional Butterworth filter (bottom-left) and artifact reduction (bottom-right). The original signal is underlayed on each window in gray. Artifact reduction decreases the effect of the corruption (presented as Mean Squared Error) much more than conventional filtering, and the signal distortion it imposes only occurs during the artifact, where the filter distorts the entire signal.

eral works have been published on using subsets of wavelet transform coefficients directly in sEMG pattern recognition systems that demonstrate an increase in classification accuracy [10], [13]. Other works have focused on using the wavelet transform to dynamically reduce noise across the time and frequency domains based on a priori knowledge about the signal and noise sources, referred to as denoising [14]-[18]. The latter approach produces a cleaned version of the original signal, providing a more direct correlation between the result and motor intention, and allowing for straightforward integration in existing systems. To the authors' knowledge, no investigation has been made into determining the feasibility of implementing a real-time signal denoising routine on a microcontroller operating independently of a PC. Demonstrating the feasibility of executing such algorithms in real-time and in an embedded system is the next logical step towards more robust pattern recognition-based control in limb prostheses.

None of these algorithms address the situation where electrodes lose their electrical coupling with the body, another potential complication on myoelectric prostheses. Little is known on the frequency of occurrence of such situations in daily use, but their effects are significant enough for leadoff event (LOE) detection circuitry to be included in many bio-potential amplifiers. Dry electrodes commonly used in prosthetic sockets are particularly susceptible to LOEs, as there is no adhesive to attach the electrodes to the skin, resulting in a complete loss of EMG often coupled with strong transients as electrodes disconnect and reconnect with the skin. LOE incidence can be reduced by making the socket fitting tighter around the stump, but this comes at the cost of comfort making it an often unacceptable compromise.

Existing classifiers that adapt to changes in signal characteristics typically react slowly and thus have limited usefulness in handling LOEs [19]. While redundancy from extra electrodes may sufficiently compensate for LOEs in some circumstances [20], Zhang et al. demonstrated that a Linear Discriminant Analysis-based classifier tolerant to electrode faults resulted in significantly increased classification accuracy [21]. Their results suggest that the signal corruption and loss of information during LOEs is a valid cause for concern even with only one out of six electrodes compromised. Their implementation used a fast retraining algorithm for the classifier that compensated for missing channels. While promising, it does not provide a generic solution applicable across different machine learning algorithms.

Pelckmans et al. [22] suggested using a probabilistic model of missing data for support vector machines that approaches mean data imputing in the case of a linear system [20]. While the research has not been applied to sEMG signals specifically, it offers a generic solution that is computationally efficient. Since this operates directly on the signal during preprocessing, it can be implemented in a modular fashion on an existing embedded system without significant modifications.

In the present study, we investigated signal denoising and data imputing to enhance the robustness of pattern recognition against noise and sensor faults during continuous sEMG classification. We evaluated classification accuracy on three common classifiers and on signal distortion with respect to denoising algorithms and demonstrated the feasibility of executing these algorithms in real-time in an embedded system for the first time. In addition, we implemented said algorithms on a low cost, low power microcontroller capable of fitting into existing prostheses, allowing for further clinical translation.

\section{Methods}

This study was approved by the Västra Götalandsregionen ethical committee (Dnr: 769-12), and written informed consent was obtained from all participants.

\section{A. Wavelet-Based Signal Denoising}

The characteristics of sEMG signals depend on many factors, but the dominant frequency band viable for wavelet analysis is in the $125-250 \mathrm{~Hz}$ range [10], with components in the $250-500 \mathrm{~Hz}$ range containing the largest relative contribution of system noise [23]. For this application, the system noise was treated as a locally-stationary additive function based on the standard deviation of the first-level $(250-500 \mathrm{~Hz})$ wavelet detail coefficients. Algorithms to reduce system noise from wavelet coefficients were selected based on reviews in previous literature with an emphasis on computational simplicity [15], [24]-[28]. Hard, soft, semi-hyperbolic (hyper), adaptive, and non-negative (non-neg) shrinkage methods were investigated in this experiment, defined in Table I. The Daubechies four tap wavelet was chosen as the mother wavelet due to its ability to effectively describe both time and frequency signal components and its low filter order [16]. The noise threshold parameter, $\lambda$, for each routine was calculated using the minimax threshold defined in (1):

$$
\lambda=\hat{\sigma} \cdot\left(0.3936+0.1829 \cdot \frac{\log (N)}{\log (2)}\right)
$$

where $\hat{\sigma}$ is the standard deviation of the system noise wavelet coefficients and $N$ is the window length in samples. This is designed to minimize the maximum mean squared error against an ideal procedure [29]. 
TABLE I

WAVELET SHRINKAGE RULES EVALUATED IN THIS EXPERIMENT.

\begin{tabular}{|c|c|}
\hline Rule & Formula \\
\hline Hard & $\hat{\gamma}_{j, i}= \begin{cases}\gamma_{j, i}, & \left|\gamma_{j, i}\right|>\lambda \\
0, & \text { otherwise }\end{cases}$ \\
\hline Soft & $\begin{array}{ll}\operatorname{sgn}\left(\gamma_{j, i}\right)\left(\left|\gamma_{j, i}\right|-\lambda\right), & \left|\gamma_{j, i}\right|>\lambda \\
0, & \text { otherwise }\end{array}$ \\
\hline Hyper & $\hat{\gamma}_{j, i}= \begin{cases}\operatorname{sgn}\left(\gamma_{j, i}\right) \sqrt{\gamma_{j, i}^{2}-\lambda}, & \left|\gamma_{j, i}\right|>\lambda \\
0, & \text { otherwise }\end{cases}$ \\
\hline Adaptive & $\hat{\gamma}_{j, i}=\operatorname{sgn}\left(\gamma_{j, i}\right)\left(\left|\gamma_{j, i}\right|-\lambda\right)+\frac{2 \lambda}{1+\exp \left(2.1 \gamma_{j, i} / \lambda\right)}$ \\
\hline Non-Neg & $\hat{\gamma}_{j, i}= \begin{cases}\gamma_{j, i}-\frac{\lambda^{2}}{\gamma_{j, i}}, & \left|\gamma_{j, i}\right|>\lambda \\
0, & \text { otherwise }\end{cases}$ \\
\hline
\end{tabular}

$\gamma_{j, i}:=$ original wavelet coefficient at level $j$ at time index $i$ $\hat{\gamma}_{j, i}:=$ de-noised wavelet coefficient at level $j$ at time index $i$

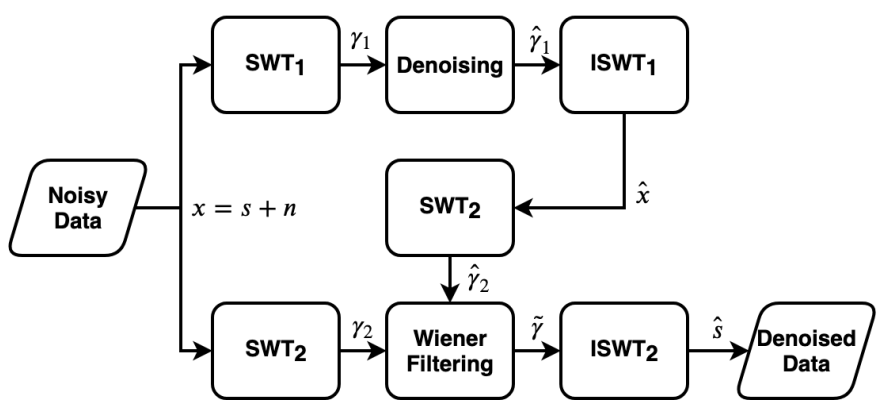

Fig. 2. Block diagram for generic wavelet-based Wiener filtering. The transforms for the Wiener coefficient estimation and for the signal filtering are separate, allowing for use of different wavelet parameters in wavelet shrinkage and Wiener filtering.

\section{B. Wiener Correction Factor}

The wavelet transform concentrates the signal energy into a relatively small number of high-valued coefficients [30]. Wavelet-based denoising then reduces the sufficiently small coefficients (assumed to be noise) towards zero, reducing the signal subspace (the number of non-zero coefficients used to describe the signal). This property, combined with the fact that the wavelet transform provides rich spectral characteristics on both the desired signal and the noise lends the process to Wiener filtering, expressed as $\tilde{\gamma}_{j, i}$ in (2).

$$
\tilde{\gamma}_{j, i}=\frac{\gamma_{j, i} \cdot \hat{\gamma}_{j, i}^{2}}{\hat{\gamma}_{j, i}^{2}+s\left(\hat{\gamma}_{1}\right)^{2}}
$$

where $s(\cdot)$ denotes the corrected sample standard deviation. This treats the desired signal and the noise as locally stationary systems, which is appropriate for sufficiently small windows on isometric contractions [31], and provides a smoother system response than wavelet shrinkage alone. The Wiener filtering coefficients can be calculated on a different transform level and mother wavelet selection than the original data, Fig. 2, but this flexibility was foregone in the current implementation to minimize processing time.

\section{Wavelet-Based Artifact Reduction}

Motion artifacts are characterized by strong, transient signal interference at low frequencies, but they have wide-band characteristics that extend into the dominant sEMG signal range [9]. A third- or fourth-order stationary wavelet transform cleanly separates the artifacts into the approximation coefficients, corresponding to the $0-62.5 \mathrm{~Hz}$ and $0-31.25 \mathrm{~Hz}$ ranges, respectively, while maintaining a one-to-one correlation of time indexes on all levels of the transform with the original signal. In this implementation, it was assumed that any sufficiently strong signal in the approximation coefficients of the transform was caused by a transient artifact. Hard thresholding was applied to each signal band (3-5) to remove the corrupted portions of the signal to produce the cleaned approximation and detail coefficients $\hat{\gamma}_{A, i}$ and $\hat{\gamma}_{D, i}$, respectively:

$$
\begin{aligned}
\theta_{k} & =\mu_{i}\left(\left|\gamma_{\mathrm{dom}}\right|\right)+k * s_{i}\left(\gamma_{\mathrm{dom}}\right) \\
\hat{\gamma}_{A, i} & = \begin{cases}\gamma_{A, i}, & \text { if }\left|\gamma_{A, i}\right|<\theta_{1} \\
0, & \text { otherwise }\end{cases} \\
\hat{\gamma}_{D, i} & = \begin{cases}\gamma_{D, i}, & \text { if }\left|\gamma_{A, i}\right|<\theta_{1} \cup\left|\gamma_{D, i}\right|<\theta_{0} \\
0, & \text { otherwise }\end{cases}
\end{aligned}
$$

where $i$ is the time index, $\gamma_{A, i}$ is the $i$-th wavelet approximation coefficient, $\gamma_{D, i}$ is the corresponding detail coefficient at decomposition level $D, \mu_{i}(\cdot)$ is the mean value operator over time, and $\gamma_{\text {dom }}$ is the wavelet decomposition level corresponding to the dominant sEMG signal frequency band. The effect of this proposed algorithm is illustrated in Fig. 1.

\section{Wavelet Processing Implementation}

At the time of the experiment, the authors were unaware of any microcontroller compatible implementation of the stationary wavelet transform (SWT) algorithm and its inverse. The appropriate routines were written in $\mathrm{C}$, leveraging the Cortex Microcontroller Software Interface Standard for optimization of filtering operations [32]. The denoising and artifact reduction routines were performed immediately prior to feature extraction on each sample window. Signals were reconstructed by recursively averaging all possible shifted, decimated inverse discrete wavelet transforms on each wavelet level, referred to as the average basis inverse [33].

Due to the nature of the wavelet transform, the operations work most efficiently on time windows with samples lengths that are a power of 2. For this work, a time window of $128 \mathrm{~ms}$ with $64 \mathrm{~ms}$ overlap sampled at $1 \mathrm{kHz}$ was selected, which falls within the typical windowing range for sEMG applications and still allows for some processing time before the control algorithm begins to feel unresponsive [1], [34]. Time performance metrics were collected for each of the proposed denoising algorithms on this window length and are shown in Fig. 3. The processing time was found to grow approximately linearly with the number of active channels and the length of the time windows. 


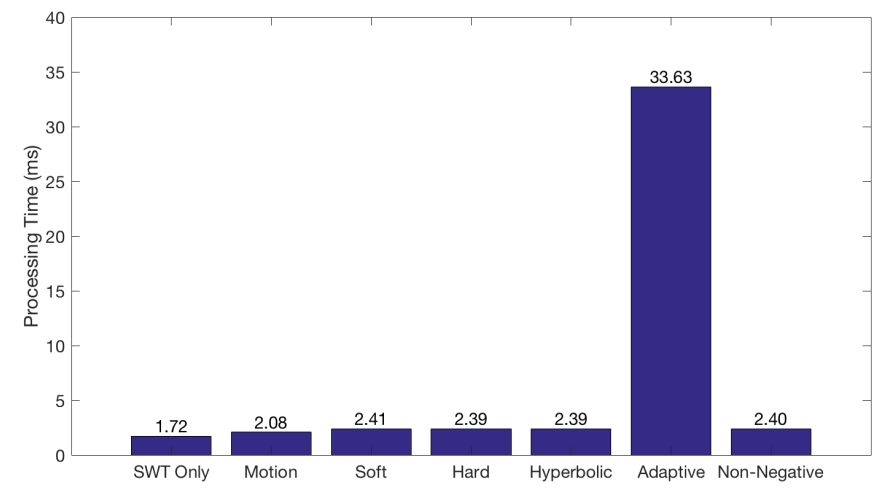

Fig. 3. Mean processing time required for each algorithm on one channel with 128 sample length windows. The label 'SWT Only' only includes the SWT and its inverse transform, 'Motion' includes the transforms and motion artifact reduction, and all others include the transforms, artifact reduction, and the listed routine.

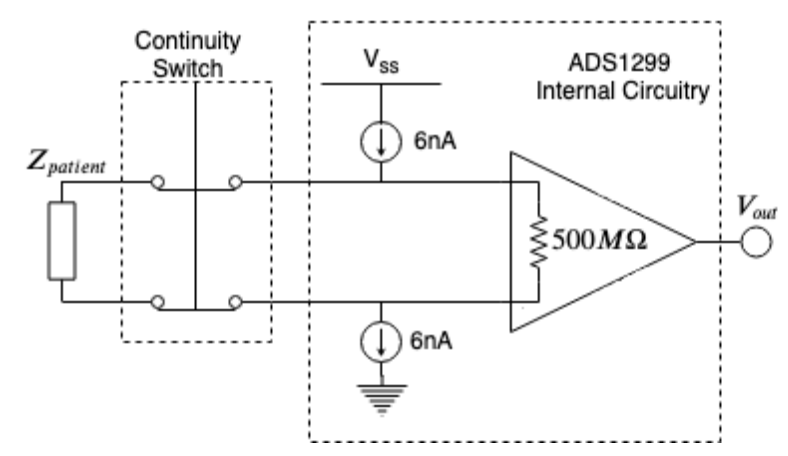

Fig. 4. Equivalent circuit for detection and simulation of lead-off events in real-time.

\section{E. Lead-Off Detection and Data Imputing Implementation}

Electrode disconnect events create a high impedance mismatch between the amplifier and the leads. The analog frontend used in this study was capable of detecting these events by adding a $6 \mathrm{nA}$ DC current source to both the positive and negative leads of each bipolar terminal and setting the input impedance for each amplifier to $500 \mathrm{M} \Omega$, illustrated in Fig. 4. During normal operation, the current is harmlessly dissipated through both the subject and the amplifier, but saturates the amplifier input when either or both leads are disconnected from the subject. Hard thresholding was applied to any signal outside the $30-70 \%$ maximum value range of the amplifier, corresponding to approximately $\pm 66 \mu \mathrm{V}$. Signals outside of this range were replaced with 0 , the mean value for EMG signals. This was not expected to increase the overall controllability of the system, but rather to decrease the chance of misclassifications resulting in movement when insufficient data is available for decoding motor intention.

\section{F. Feature Extraction and Classification}

The Time Domain feature set proposed by Hudgins et al. contains some of the most commonly investigated features in EMG applications due to their low computational complexity and high descriptiveness [35]. In addition, a relative comparison of these and other common features suggests that this set (composed of mean absolute value (MAV), zero crossings (ZC), waveform length (WL), and signed slope change (SSC)) is adequate for MPR [36], [37].

The processing and memory requirements of many pattern recognition systems limits the selection for real-time embedded applications. Multi-Layer Perceptron (MLP), Support Vector Machine (SVM), and Linear Discriminant Analysis (LDA) are all commonly used for EMG classification [38] and are capable of real-time implementation in an embedded system. Multi-class support was implemented for the SVM classifier using a "one-vs-all" scheme. The MLP classifier used one layer of 16 hidden neurons using the hyperbolic tangent activation function and the softmax activation function on the output neurons. The NetLab 3.3 Neural Network library was used for MLP classifier training on a PC [39]. The signal detection threshold was calculated using the average MAV feature value across all rest signal windows in the training data. Any time windows with a MAV feature smaller than this value bypassed the classifier and were considered a rest state.

\section{G. Training Protocol}

The training data sets for the pattern recognition algorithms in all experiments and for all movements consited of recordings of three second contractions repeated three times, and each separated by three seconds of rest. Signal recordings were taken from four sets of pre-gelled $\mathrm{Ag}-\mathrm{AgCl}$ electrodes (GS26, Bio-Medical Instruments, USA) placed in bipolar configurations with approximately equal spacing around the proximal third of each subject's dominant forearm. Subjects were asked to perform contractions at 70-80\% of their maximum voluntary contraction strength. Inactive and lowlevel transient portions of each contraction were discarded such that only the center $70 \%$ was preserved, a value that has been empirically found to conserve isometric and part of the dynamic portions of muscle contractions in healthy subjects [40]. Data for training the rest classification and floor noise were obtained from the center $50 \%$ of each of the rest periods in the recordings. A rest state class was included to ensure small signals that exceed the floor noise threshold were not automatically assumed to indicate intended movement. EMG data were separated and concatenated into arrays corresponding to the signal in each movement. The data arrays were then windowed, and signal features calculated from those windows were used to construct training, testing, and validation sets for the classifiers. Visual cueing for contractions, signal recording, and feature extraction for classifier training were all performed using BioPatRec running on MATLAB 2016b [40].

\section{H. Experiment I. Offline Wavelet Parameter Selection}

The sEMG recordings for evaluation and selection of appropriate wavelet parameters were obtained from a publicly accessible data set of 20 subjects performing 10 wrist, hand, and forearm movements recorded using the methodology described in the Training Protocol section [41]. Data were originally sampled at $2 \mathrm{kHz}$, but were decimated to $1 \mathrm{kHz}$ and the four movements not used in the real-time analysis were discarded. The hand open and close, wrist flexion and 
extension, and forearm pronation and supination movements were used for analysis. Classifier training data was composed of a random selection of $90 \%$ of the feature windows in each movement, and testing and validation data was pooled together in the remaining $10 \%$. 10-fold data cross-validation was used to generalize the classifier performance results.

After filtering power line noise, the dominant noise sources for sEMG signals at rest are from the electrode-skin interface, the environment, and the amplifier itself [9]-[11]. Noise from the amplifier used can be modeled as roughly Gaussian white noise in the frequency range of interest [42]. Noise from the electrode-skin interface has a pink noise spectrum with the amplitude approaching that of the amplifier near 200$300 \mathrm{~Hz}$ [11]. To the authors' knowledge, little investigation has been made into its probability distribution, so it was assumed to be Gaussian. Environmental noise can depend on many factors, making it infeasible to model within the scope of this experiment, so no attempt was made to simulate it. Based on this information and the use of a $20 \mathrm{~Hz}$ high-pass filter in the amplifier (reducing the amplitude of the electrode interference at low frequencies), poor signal quality was modeled using white Gaussian noise. Pseudo-random Gaussian noise was added to each movement in the testing data and was scaled to $20 \%$ of one standard deviation of the raw signal amplitude for the respective movement.

Four metrics were used to compare the performance of each of the wavelet-based signal processing algorithms: global accuracy, the Mean Squared Error (MSE) between the original data and the filtered original data $\left(\mathrm{MSE}_{\mathrm{rec}}\right)$, the MSE between the filtered noisy data and the original data $\left(\mathrm{MSE}_{\mathrm{ref}}\right)$, and the MSE between the filtered noisy data and the filtered original data $\left(\mathrm{MSE}_{\text {noise }}\right)$. To reduce the burden of subject testing to a reasonable level, only the best performing model in this test was considered for the remainder of the experiment.

\section{Experiment II. Real-Time}

Nine able-bodied subjects between the ages of 22 and 29 $(\mu=25, \mathrm{SD}=2.5)$ volunteered for the work. Real-time sEMG signals were collected from subjects using the methodology described in the Training Protocol section. EMG signals were sampled at $2 \mathrm{kHz}$ with 24-bit resolution and $24 \mathrm{~V} / \mathrm{V}$ gain using an ADS_BP signal acquisition unit [43]. The sEMG signals were then decimated to $1 \mathrm{kHz}$ and filtered using a second order $20 \mathrm{~Hz}$ IIR high-pass filter and a $50 \mathrm{~Hz}$ IIR notch filter. The resulting data from these sessions and from the Motion Tests described below were made publicly available on GitHub [41].

To evaluate the performance of the proposed wavelet processing routine, subjects were instructed to perform two Motion Tests, described in the section Real-Time Performance Evaluation, with one Motion Test relying solely on the $20 \mathrm{~Hz}$ high-pass and $50 \mathrm{~Hz}$ notch filters, and one with the addition of wavelet processing operating on the fourth-level transform. Subjects were instructed to start each contraction with their elbow resting on the table and the forearm raised such that no leads were touching the table. They were then instructed to bump their wrist against the table once on each side of a small obstacle, pivoting on their elbow, once during each

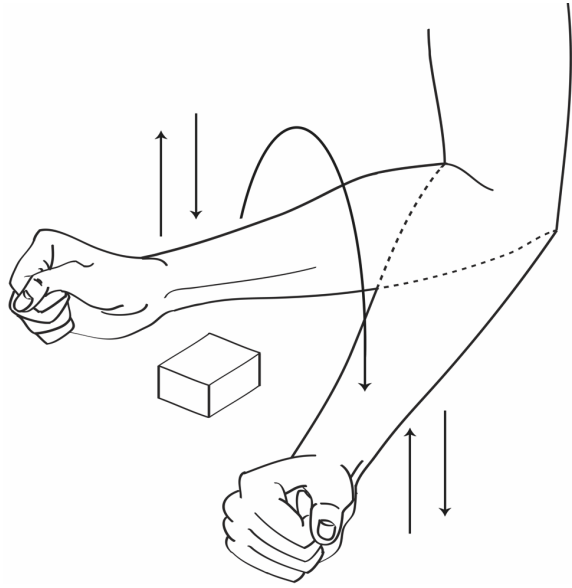

Fig. 5. Motion artifacts were generated in real-time by having the subject bump their wrist against the table on either side of a small obstacle during each contraction [44]

contraction, illustrated in Fig. 5. This action was intended to simulate the typical case for sEMG signal transients where the user may bump the prosthetic against an object or shift the appendage in the socket or electrode band.

To test the efficacy of the lead-off detection and handling subsystem, two Motion Tests were performed, one with mean data imputing enabled, and one without any extra processing. A single-pole double-throw continuity switch was attached to each lead pair, Fig. 4, and operated manually using a random number generator to indicate disconnect events. The generator indicated new events at a pseudo-random interval with a two second mean time interval between events with a standard deviation of one second. Disconnect events lasted for between approximately 0.2 and 0.5 seconds, and began occurring immediately after starting each test.

\section{J. Experiment III. Simulated Real-Time}

To observe the effect of a higher number of signal corruption episodes, the proposed routines were tested on a series of pre-recorded Motion Tests with artificially added noise and sensor faults. This was done by feeding previously recorded sEMG signals from Motion Tests into the microcontroller and recording the resulting classifications. Standard offline analysis of EMG data is often an unreliable indicator of realtime performance, likely due to the loss of feedback and the sequential nature of the data [45]. Using pre-recorded Motion Tests still distorts the feedback effect, due to changes in signal and processing, but maintains the structure and order of the sEMG signals.

The simulated tests were performed on a separate data set where sEMG signals were recorded from the left arms of 15 able-bodied subjects performing 10 wrist and forearm movements using the methodology described in the Training Protocol section [40]. This data set was recorded at $2 \mathrm{kHz}$ with a second order digital high-pass filter at $20 \mathrm{~Hz}$ and a notch filter centered at $50 \mathrm{~Hz}$. It also contained sEMG data from both the pattern recognition training and the full Motion Tests. As the EMG data for the tests were recorded using different time 


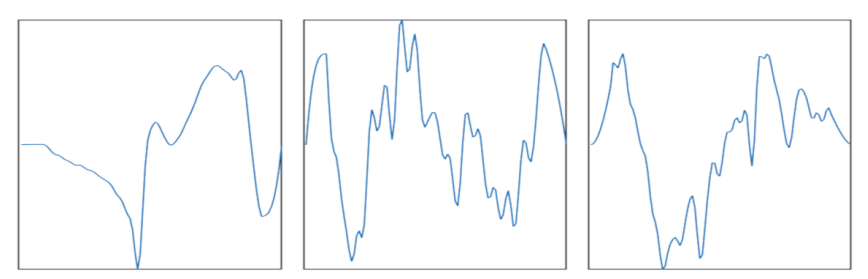

Fig. 6. Examples of contact motion during rest (left panel), contact motion during contraction (middle panel), and cable motion (right panel).

window parameters, only the first $128 \mathrm{~ms}$ in each time window, after decimating to $1 \mathrm{kHz}$, was extracted to form the training and testing sets. This made it possible to send the recordings to the embedded system for processing while maintaining as much of the original structure of the data as possible.

The EMG data used for comparing wavelet processing with conventional filtering were modified with a set of previously recorded motion artifacts [15]. Artifacts were originally recorded at $2 \mathrm{kHz}$ using $\mathrm{Ag}-\mathrm{AgCl}$ bipolar electrodes and were generated by tapping the electrodes, cable movement, and rapid arm movement while the subjects' muscles were at rest. Motion artifact magnitude was experimentally determined to extend up to 150 times the standard deviation of normal EMG signals, with smaller perturbations mimicking real-life scenarios resulting 1 to 10 times EMG standard deviation amplitude. The artifacts, examples of which are shown in Fig. 6, were decimated and filtered to match the properties of the test EMG signal. For each time window in the Motion Test, random artifacts were added on at least one channel (chosen at random) at random offsets with magnitudes corresponding to between 1 and 10 times the standard deviation of the signal strength of that window. Wavelet processing, when applicable, and pattern classification were performed on the microcontroller using a pre-trained LDA classifier by providing each time window over a serial connection and reading the resulting classifications. This test included wavelet-based artifact reduction using the third- and fourth-level transforms in addition to conventional filtering.

Noise recorded from a set of disconnected leads using the same setting as the Motion Tests was used as the data source for simulating lead-off events. The noise was then decimated to $1 \mathrm{kHz}$ and separated into $128 \mathrm{~ms}$ time windows. Motion Test data on at least one channel (selected at random) on each time window were replaced with either a random sample of recorded noise (for conventional handling) or zeros (for mean data imputing).

Neither artifacts nor lead-off events were simulated for the training data, but to compensate for the non-linear effects wavelet processing has on the data, the appropriate routine was applied to both the training and testing data for that case, illustrated in Fig. 7. The ground truth for the simulated tests in both cases was determined by running the classifier against the recorded Motion Tests without simulated LOEs or motion artifacts and saving the positions of the correct classifications. Any incorrect predictions made in this situation were ignored, as the proposed algorithms were not expected to significantly increase the predictive power of the classifiers on clean data

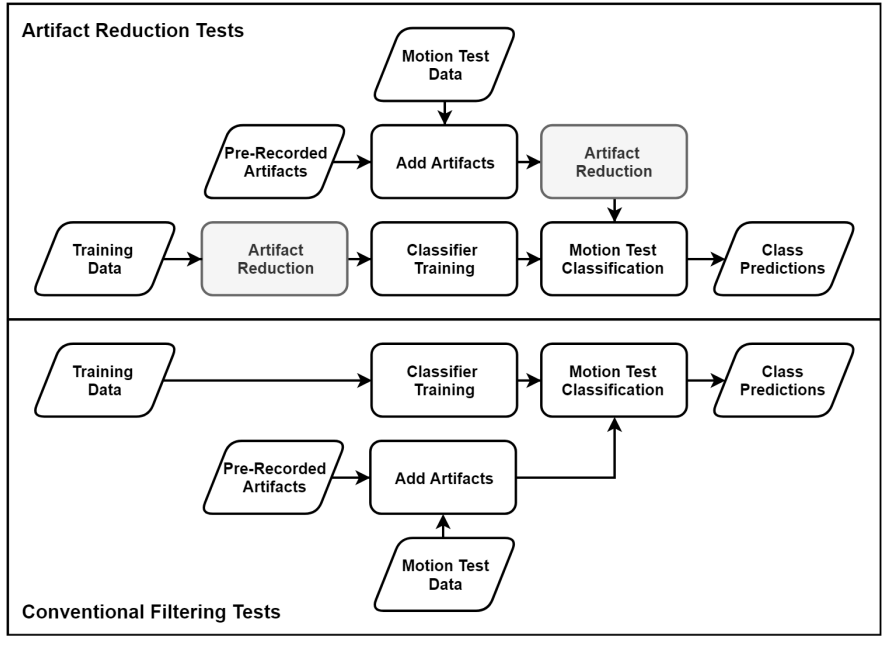

Fig. 7. Block diagram showing the training and testing procedure used to evaluate wavelet-based motion artifact reduction on pre-recorded Motion Tests.

sets.

\section{K. Real-Time Performance Evaluation (Experiments II-III)}

A modified version of Kuiken et al.'s Motion Test [46] was used to generate data for the real-time evaluation used in this work. Subjects were visually cued to perform trials of three random permutations of the movement set for each test. They were asked to hold the cued contractions at 70$80 \%$ of maximum voluntary contraction strength until the system made 20 correct predictions or for up to 10 seconds. Signal recording, data visualization, classifier training, and visual cueing were all controlled using the BioPatRec software suite running on MATLAB 2016b [40]. The mean per-class accuracy, specificity, sensitivity, precision, and false activation error, described below, across all classes were used as performance metrics for all tests in addition to completion time, selection time, and completion rate. The completion time and completion rate were not calculated for the simulated experiments, as the biofeedback the Motion Tests rely on was lost when the signal processing algorithm was changed. Specificity, sensitivity, and precision metrics were included to compensate for the inherent bias of global accuracy [45], [47].

The conventional calculation for error rates in multi-class systems weight all misclassifications equally, and therefore it is not useful for measuring the likelihood that a system will reject a classification based on uncertainty or missing data values. In order to account for this, we employed an additional metric referred to in this work as the False Activation Error (FAE), defined as the percentage of class-wise false negative misclassifications resulting in movement. This calculation is similar to the standard class-wise error rate, but does not penalize "rest" misclassifications. FAE was originally proposed by Hargrove et al. [12] to accommodate for the disproportionately detrimental effects of misclassifications resulting in unintended movements. This extra metric was used to show the difference in unintended movements resulting from LOEs and motion artifacts produced by the proposed algorithms. For instance, if 

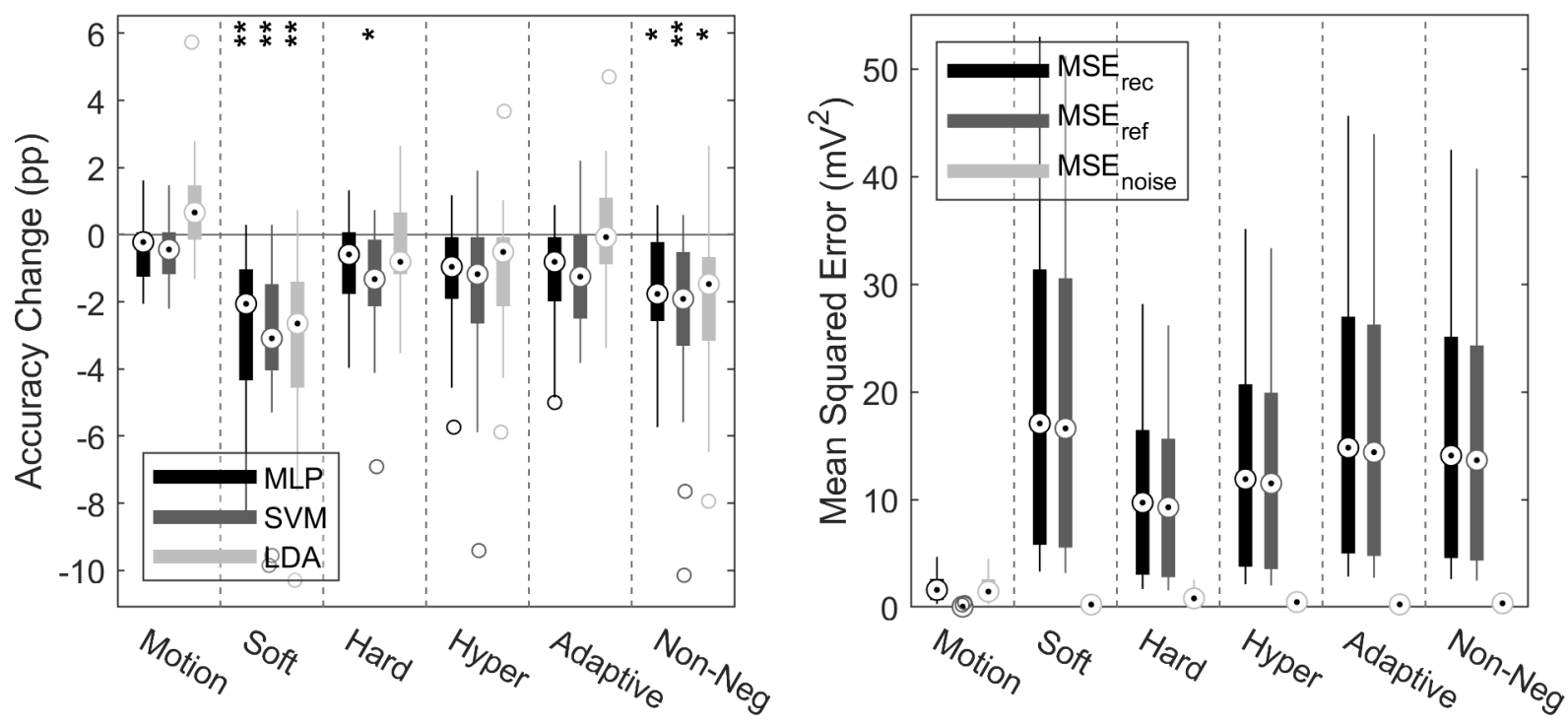

Fig. 8. Experiment I. Offline accuracy change (left) and Mean Squared Error comparison (right) of wavelet-based processing vs. conventional processing. Data shown are median values (circle with dot), inter-quartile range (thick bar), and data range (thin bar), with hollow circles as data outliers. Motion artifact reduction using LDA classifier showed the only apparent accuracy improvement ( $n s$ ).

an LOE occurred during a close hand movement, the system would ideally reject data from the affected electrodes, reducing the chance of interpreting an unintended movement, such as open hand or wrist rotation, until the electrodes regained connectivity. Wilcoxon signed rank tests were used on subjectspecific means on each metric independently for statistical analysis using Bonferroni correction to adjust for multiple comparisons.

Classifier training and artifact reduction on the training data were performed on a PC. All other processing steps for the testing data, including digital filtering, wavelet-based processing, feature extraction, and pattern classification, were implemented on the microcontroller to allow for independent and mobile operation in prosthetic devices. Pattern recognition algorithms were trained using a data set recorded prior to each set of comparative tests. Embedded processing was performed on a Texas Instruments ARM Cortex-M4 processor; a full description of the hardware can be found in [43].

\section{RESULTS}

\section{A. Wavelet Parameter Selection}

Motion artifact reduction with the LDA classifier without wavelet denoising was the only set of parameters that indicated any numerical global accuracy improvement over conventional filtering $(n s)$, Fig. 8 left panel. This configuration also showed the smallest signal distortion for the reference signal compared to the noisy signal, indicating it would have the most positive effect on reduction of signal corruption, Fig. 8 right panel.

\section{B. Real-Time Test Results}

Real-time test results between conventional filtering and wavelet-based artifact reduction showed no significant differences, Fig. 9a. Real-time data imputing tests showed a decrease in precision $(p<.05)$ of 12.0 percentage points (pp), but showed no other significant differences between data imputing and conventional filtering, Fig. 9b. A full summary of the results is reported in Table II.

\section{Simulation Test Results}

The third-level wavelet processing performed best on all metrics in simulation tests except selection time with an improvement in accuracy over conventional filtering by $1.80 \mathrm{pp}$ $(p<.001)$, in sensitivity by $2.90 \mathrm{pp}(p<.001)$, in specificity by $1.9 \mathrm{pp}(p<.001)$, in FAE by $22.9 \mathrm{pp}$ $(p<0.001)$, and in precision by $5.00 \mathrm{pp}(p<.001)$, Fig. 9a. The third-level transform increased the selection time by $327 \mathrm{~ms}(p<.01)$, indicating that the system took longer to register that a correct movement was being attempted by a significant degree in the presence of artifacts. The fourthlevel transform showed an increase in accuracy by $1.20 \mathrm{pp}$ $(p<.001)$, in specificity by $1.30 \mathrm{pp}(p<.001)$, and FAE by $16.6 \mathrm{pp}(p<.001)$, with no significant change in sensitivity, precision, or selection time. Data imputing showed an improvement in accuracy of $4.50 \mathrm{pp}(p<.001)$, in specificity of $6.00 \mathrm{pp}(p<.001)$, in precision of $3.40 \mathrm{pp}$ $(p<.05)$, and in FAE by $54.3 \mathrm{pp}(p<0.001)$. Data imputing showed reduced performance in sensitivity of $9.33 \mathrm{pp}$ $(p<.001)$ and in selection time by $906 \mathrm{~ms}$, Fig. 9b. A full summary of the results is reported in Table II.

\section{DISCUSSION}

Phinyomark et al.'s work on wavelet denoising showed significant noise reduction in EMG signals, but the investigation was limited to the MAV feature, leaving its effect on other signal features in question [16]. The only literature found investigating the effect of denoising with short time windows on overall classifier accuracy only showed a relatively small improvement for the MLP classifier and was implemented 


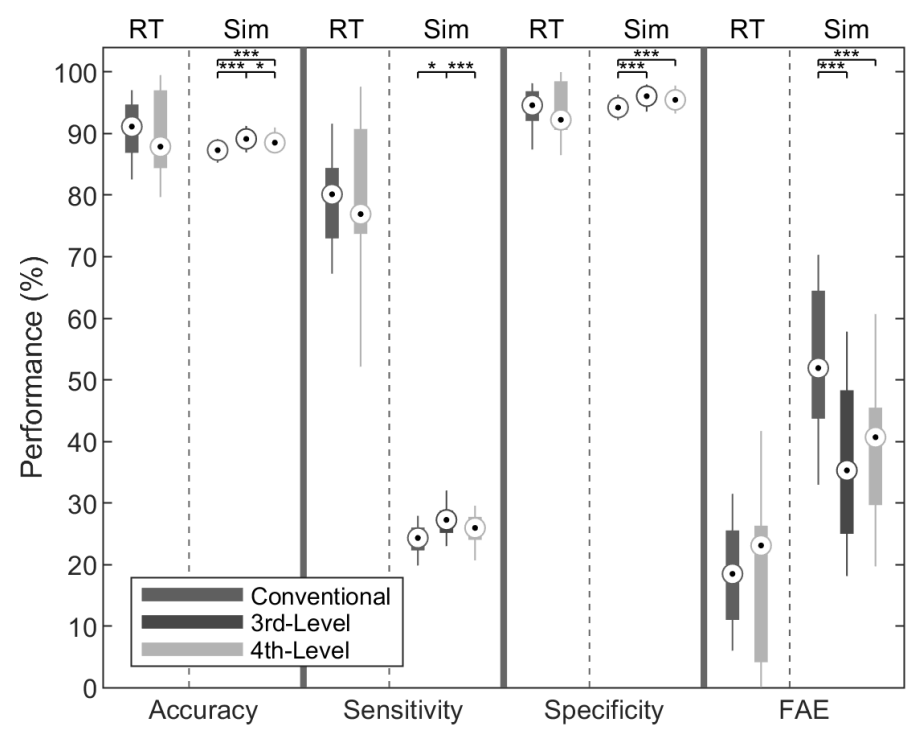

a) Artifact Reduction Results

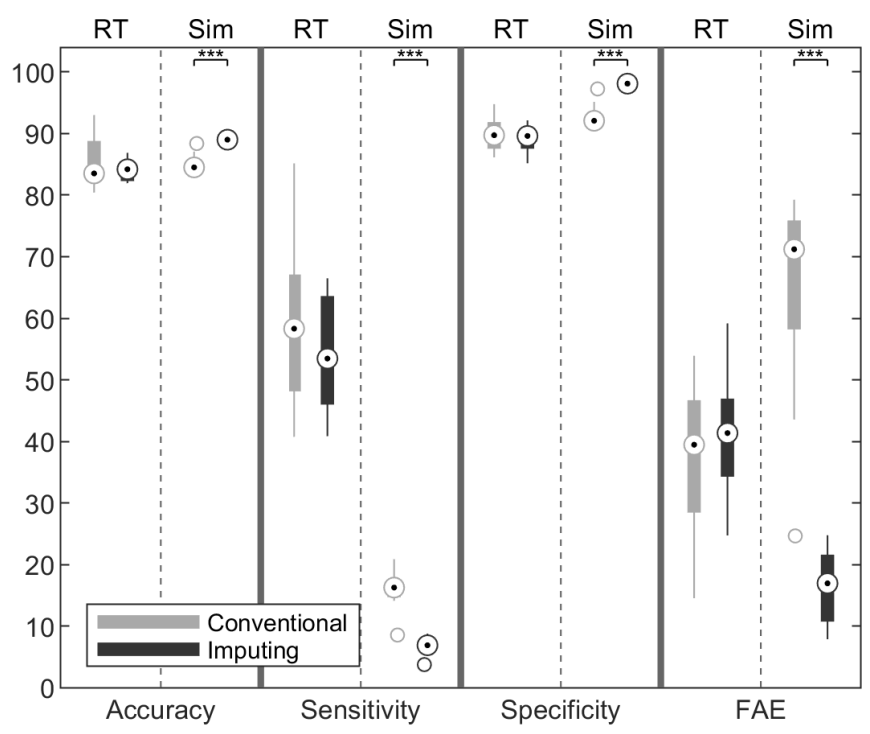

b) Data Imputing Results

Fig. 9. Box plot of real-time (RT) and simulated real-time (Sim) metrics comparing the proposed wavelet-based motion artifact reduction (a) and data imputing (b) routines with conventional signal processing on an embedded system. Data shown are values (circle with dot), inter-quartile range (thick bar), and data range (thin bar), with hollow circles as data outliers. The wavelet processing algorithm used third- and fourth-level transforms, meaning motion artifacts were assumed to have their dominant energy in the $0-62.5 \mathrm{~Hz}$ and $0-31.25 \mathrm{~Hz}$ frequency bands, respectively.

TABLE II

SUMMARY OF REAL-TIME AND SIMULATED RESULTS. VALUES ARE MEDIANS (IQR).

\begin{tabular}{|c|c|c|c|c|c|}
\hline & \multicolumn{3}{|c|}{ Artifact Reduction } & \multicolumn{2}{|c|}{ Data Imputing } \\
\hline & Conventional & Fourth Level & Third Level & No Imputing & Mean Imputing \\
\hline \multicolumn{6}{|l|}{ Real-Time $(\mathrm{n}=9)$} \\
\hline Accuracy $(\%)$ & $91.1(7.90)$ & $87 . \overline{(12.6)}$ & & $83.5(6.59)$ & $84.2(3.07)$ \\
\hline Sensitivity (\%) & $80.1(11.5)$ & $76.9(17.0)$ & & $58.3(19.0)$ & $53.5(17.7)$ \\
\hline Specificity (\%) & $94.6(4.83)$ & $92.2(7.92)$ & & $89.7(4.34)$ & $89.6(2.69)$ \\
\hline Precision $(\%)$ & $69.4(7.08)$ & $80.1(20.4)$ & & $47.9(11.5)$ & $35.9(6.18) *$ \\
\hline Completion Rate (\%) & $61.1(21.5)$ & $58.3(16.0)$ & & $36.1(15.3)$ & $38.9(20.8)$ \\
\hline Selection Time (ms) & $706(695)$ & $828(388)$ & & $2020(1100)$ & $1980(1660)$ \\
\hline Completion Time (ms) & $3110(1080)$ & $3100(2100)$ & & $3610(2030)$ & $3040(1960)$ \\
\hline False Activation (\%) & $18.5(14.5)$ & $23.1(22.2)$ & & $39.5(18.3)$ & $41.3(12.7)$ \\
\hline \multicolumn{6}{|l|}{ Simulated $(\mathrm{n}=15)$} \\
\hline Accuracy $(\%)$ & $87.3(2.13)$ & $88 . \overline{5}(1.97)$ *** & $89 . \overline{1}(\overline{2} . \overline{5} 2) * * *$ & $\overline{8} 4 . \overline{5}(1.63)$ & $89 . \overline{0}(\overline{0} . \overline{8} 10) \bar{*} * \bar{*}$ \\
\hline Sensitivity (\%) & $24.3(3.72)$ & $25.9(3.74)$ & $27.2(3.64) *$ & $16.2(2.86)$ & $6.87(1.71) * * *$ \\
\hline Specificity (\%) & $94.2(2.31)$ & $95.5(1.76) * * *$ & $96.1(2.59) * * *$ & $92.1(1.97)$ & $98.1(1.21) * * *$ \\
\hline Precision $(\%)$ & $48.9(7.92)$ & $48.9(15.6)$ & $53.9(18.3) * * *$ & $38.9(7.16)$ & $42.3(6.17) *$ \\
\hline Selection Time (ms) & $186(198)$ & $423(311)$ & $513(377) * *$ & $83.9(76.6)$ & $990(415) * * *$ \\
\hline False Activation (\%) & $51.9(20.8)$ & $40.7(15.8) * * *$ & $35.3(23.3) * * *$ & $71.2(17.7)$ & $16.9(10.9) * * *$ \\
\hline
\end{tabular}

(*) indicates statistical significance from null hypothesis at $p<.05$

(**) indicates statistical significance from null hypothesis at $p<.01$

$(* * *)$ indicates statistical significance from null hypothesis at $p<.001$

with more neurons than considered feasible for real-time implementation in this experiment [15]. Wavelet-based denoising may have an unseen positive effect on noise sources that more closely match real-world use, but our experiments showed it degraded performance compared to conventional filtering when presented with sEMG signals corrupted with Gaussian noise. Additionally, the current investigation used pre-gelled $\mathrm{Ag}-\mathrm{AgCl}$ electrodes, which have different electrical characteristics than stainless-steel dry electrodes typically seen in commercial prostheses. While motion artifacts and LOEs likely exhibit the same signal properties using either electrode material, recordings taken from dry electrodes may include additional noise that would be removed via the proposed signal denoising routine. This limitation will be addressed in future work. The processing time for wavelet-based signal processing was found to increase roughly linearly with the number of active channels, which potentially limits its applicability in real-time processing on systems with a high number of 
channels. However, the added delay was not sufficient enough to noticeably degrade system performance in this application.

The real-time experiments showed little effect from either artifact reduction or data imputing. This suggests either that the protocol used in the real-time portion of the present work generated an insufficient number of signal artifacts to simulate real-life scenarios or that signal artifacts do not present a significant problem in real-life scenarios. The motion artifacts corrupting the EMG signals in the Motion Tests were on the order of $100 \mathrm{~ms}$. With a window overlap of $64 \mathrm{~ms}$, up to six windows could have been corrupted by motion artifacts from hitting the table both times, plus any that occurred during the transit of the forearm above the table. Given that an evaluation per movement lasts up to 10 seconds (approximately 150 time windows), the motion artifacts could only affect a small portion of the total predictions. This was insufficient to cause a significant difference in the Motion Test outcomes, and hence the need of the simulated experiments where the signals were artificially corrupted more frequently. Similar logic applies to the small difference found when comparing conventional filtering to mean data imputing, as the lead-off events lasted between 0.2 and 0.5 seconds. In daily use and out of controlled environments, the number of episodes in which such artifacts could frustrate the user is unknown and difficult to estimate. Factors related to prosthetic fitting, such as hardware, activity level, and stump condition, would influence susceptibility to artifacts and their incidence. These factors were not investigated in the current work, but an investigation into the error and False Activation Error caused by signal artifacts in daily life activities and their effects on perceived system performance will be investigated in future work.

The simulated experiments were expected to highlight any changes too subtle to see in the real-time tests. Artifact reduction using a third-level wavelet transform showed better performance than the fourth-level transform along some metrics $(p<.05)$, indicating frequency components in the motion artifacts extended past the $31.25 \mathrm{~Hz}$ boundary addressed by the fourth-level approximation coefficients. Clancy et al. suggested cable motion artifacts can extend up to around $50 \mathrm{~Hz}$ [48], which was corroborated by our results. This may partially explain the lack of improvement seen in the real-time experiment, as only the fourth-level transform was tested. Using a decreased transform order also reduced the computational complexity and memory requirements of the artifact reduction routine, but further increased the selection time. Results for the simulated data imputing tests were more mixed, showing an improvement in performance regarding accuracy and specificity, but decreased sensitivity and slower selection time. The results also showed the dramatic improvement in the False Activation Error, indicating that the number of misclassifications resulting in unintended movements was effectively reduced, but at the cost of a significant increase in the selection time. However, given that LOEs and motion artifacts are typically short-term signal corruption sources in prosthetic sockets, the reduced responsiveness indicated by the selection time is unlikely to significantly hamper the overall controllability. Based on the slower selection time and the higher FAE, both algorithms appear to bias the classifier towards making either the ground truth movement or no movement at all for the duration of the signal corruption events. These results suggest that mean data imputing is a potentially useful strategy for handling LOEs during continuous sEMG classification, given that a short-term reduction in responsiveness is often preferable to unintended movements.

Increases in most of the performance metrics in the simulated experiments came at the cost of a significant increase in selection time for both data imputing and artifact reduction, reducing the system responsiveness during channel corruption. It is noteworthy to emphasize that at least one channel was corrupted with a lead-off event or motion artifact on every time window. As such, these results serve as a worst-case scenario rather than a typical use-case, and as seen in the offline analysis results, neither method had a significant impact on the signal when no artifacts or noise were present. Based on the results, the proposed algorithms would tend to produce no actions during periods of fast limb movements, when the stump shifts in the socket, or when a load is applied to the end of the prosthetic. In each of these scenarios, producing no movements is likely the desired outcome. More investigation needs to be done on how the proposed algorithms affect the controllability and responsiveness of prosthetic devices in reallife environments.

Having an effective implementation of artifact reduction and data imputing on a mobile processing platform allows for investigation into their effects in real-life prosthetic use. We have previously developed an evaluation method using an embedded system in which the subject can report perceived misclassification while operating the prosthesis in daily life and for long periods of time [49]. The Assessment for Capacity of Myoelectric Control [50], the Activities Measure for Upper Limb Amputees [51], and the Southampton Hand Assessment Procedure [52] all provide insight into prosthetic controllability with respect to functional tasks simulating realworld environments, but they are cross-sectional in nature (a single point in time) and are not performed out in the real world. These assessments could be performed using a wireless interface to a base station for all the required processing without interfering with the subject mobility. However, such strategy cannot be used in daily life where the subject will encounter variable sources of noise and face motion artifacts owning to different activities. Further work will focus on evaluating the present algorithms in such circumstances.

\section{CONCLUSION}

In this work, we investigated the feasibility and effectiveness of implementing wavelet-based signal processing and data imputing for continuous sEMG classification on a self-contained prosthetic system. We proposed a novel and efficient method for EMG signal imputing and modifications to existing wavelet denoising and artifact reduction routines to allow for their implementation on a wearable prosthesis. Wavelet denoising proved ineffective for removing wide-band, Gaussian noise. Real-time tests failed to show a significant effect using the proposed routines, but increasing the rate of errors in simulated 
tests highlighted the benefits and caveats of the routines. Simulated tests showed significant increases in many performance metrics (namely the reduction of erroneous movements), but also showed a large increase in selection time, reducing the system responsiveness during channel corruption events. Having these systems implemented for real-time classification on a self-contained prosthesis reduces the barrier for more realistic assessment, and it could help bring pattern recognition-based prosthetic devices to wider clinical implementation.

\section{ACKNOWLEDGMENT}

The authors would like to thank the volunteers who participated in this study. The authors had full access to all of the data, devices, and materials used in this study and take complete responsibility for the integrity of the data and the accuracy of the data analysis and interpretation of outcomes.

\section{REFERENCES}

[1] M. Asghari Oskoei and H. Hu, "Myoelectric control systems-A survey," Biomedical Signal Processing and Control, vol. 2, no. 4, pp. 275-294, 2007.

[2] F. Cordella, A. L. Ciancio, R. Sacchetti, A. Davalli, A. G. Cutti, E. Guglielmelli, and L. Zollo, "Literature review on needs of upper limb prosthesis users," Frontiers in Neuroscience, vol. 10, no. MAY, pp. 1-14, 2016.

[3] B. Peerdeman, D. Boere, H. Witteveen, R. H. in 't Veld, H. Hermens, S. Stramigioli, H. Rietman, P. Veltink, and S. Misra, "Myoelectric forearm prostheses: state of the art from a user-centered perspective." Journal of Rehabilitation Research and Development, vol. 48, no. 6, pp. 719-37, 2011.

[4] E. Biddiss, D. Beaton, and T. Chau, "Consumer design priorities for upper limb prosthetics," Disability and Rehabilitation: Assistive Technology, vol. 2, no. 6, pp. 346-357, 2007.

[5] P. Gallagher, D. Desmond, and M. MacLachlan, Psychoprosthetics Springer Science \& Business Media, 2007.

[6] K. Østlie, I. M. Lesjø, R. J. Franklin, B. Garfelt, O. H. Skjeldal, and P. Magnus, "Prosthesis rejection in acquired major upper-limb amputees: a population-based survey," Disability and Rehabilitation: Assistive Technology, vol. 7, no. 4, pp. 294-303, 2012.

[7] A. Fougner, Ø. Stavdahl, P. J. Kyberd, Y. G. Losier, P. A. Parker, and S. Member, "Control of Upper Limb Prostheses: Terminology and Proportional Myoelectric Control-A Review," IEEE Transactions on Neural Systems and Rehabilitation Engineering, vol. 20, no. 5, pp. 663677, 2012

[8] D. Farina, N. Jiang, H. Rehbaum, A. Holobar, B. Graimann, H. Dietl, and O. C. Aszmann, "The extraction of neural information from the surface EMG for the control of upper-limb prostheses: Emerging avenues and challenges," IEEE Transactions on Neural Systems and Rehabilitation Engineering, vol. 22, no. 4, pp. 797-809, 2014.

[9] C. J. De Luca, L. D. Gilmore, M. Kuznetsov, and S. H. Roy, "Filtering the surface emg signal: Movement artifact and baseline noise contamination," Journal of Biomechanics, vol. 43, no. 8, pp. 1573-1579, 2010.

[10] M. Reaz, M. Hussain, F. Mohd-Yasin et al., "Techniques of emg signal analysis: detection, processing, classification and applications," Biological Procedures Online, vol. 8, no. 1, pp. 11-35, 2006.

[11] E. Huigen, A. Peper, and C. Grimbergen, "Investigation into the origin of the noise of surface electrodes," Medical and biological engineering and computing, vol. 40, no. 3, pp. 332-338, 2002.

[12] L. J. Hargrove, E. J. Scheme, K. B. Englehart, and B. S. Hudgins, "Multiple binary classifications via linear discriminant analysis for improved controllability of a powered prosthesis," IEEE Transactions on Neural Systems and Rehabilitation Engineering, vol. 18, no. 1, pp. 49-57, 2010.

[13] K. Englehart, B. Hudgin, and P. A. Parker, "A wavelet-based continuous classification scheme for multifunction myoelectric control," IEEE Transactions on Biomedical Engineering, vol. 48, no. 3, pp. 302-311, 2001.
[14] D. Pani, G. Barabino, L. Citi, P. Meloni, S. Raspopovic, S. Micera, and L. Raffo, "Real-time neural signals decoding onto off-the-shelf dsp processors for neuroprosthetic applications," IEEE Transactions on Neural Systems and Rehabilitation Engineering, vol. 24, no. 9, pp. 9931002, Sep. 2016.

[15] J. Maier, A. Naber, and M. Ortiz-Catalan, "Improved prosthetic control based on myoelectric pattern recognition via wavelet-based de-noising," IEEE Transactions on Neural Systems and Rehabilitation Engineering, vol. 26, no. 2, pp. 506-514, 2018.

[16] A. Phinyomark, C. Limsakul, and P. Phukpattaranont, "A comparative study of wavelet denoising for multifunction myoelectric control," in International Conference on Computer and Automation Engineering. IEEE, 2009, pp. 21-25.

[17] P. Mithun, P. C. Pandey, T. Sebastian, P. Mishra, and V. K. Pandey, "A wavelet based technique for suppression of emg noise and motion artifact in ambulatory ecg," in IEEE International Conference on Engineering in Medicine and Biology Society. IEEE, 2011, pp. 7087-7090.

[18] A. Akansu, W. Serdijn, and I. Selesnick, "Wavelet transforms in signal processing: a review of emerging applications," Physical Communication, vol. 3, no. 1, pp. 1-18, 2010.

[19] S. Jain, G. Singhal, R. J. Smith, R. Kaliki, and N. Thakor, "Improving long term myoelectric decoding, using an adaptive classifier with label correction," in IEEE International Conference on Biomedical Robotics and Biomechatronics, June 2012, pp. 532-537.

[20] P. J. García-Laencina, J.-L. Sancho-Gómez, and A. R. Figueiras-Vidal, "Pattern classification with missing data: a review," Neural Computing and Applications, vol. 19, no. 2, pp. 263-282, 2010.

[21] X. Zhang and H. Huang, "A real-time, practical sensor fault-tolerant module for robust EMG pattern recognition," Journal of NeuroEngineering and Rehabilitation, vol. 12, no. 1, p. 18, 2015.

[22] K. Pelckmans, J. De Brabanter, J. A. Suykens, and B. De Moor, "Handling missing values in support vector machine classifiers," Neural Networks, vol. 18, no. 5, pp. 684-692, 2005.

[23] G. Li, Y. Li, L. Yu, and Y. Geng, "Conditioning and sampling issues of emg signals in motion recognition of multifunctional myoelectric prostheses," Annals of Biomedical Engineering, vol. 39, no. 6, pp. 17791787, 2011.

[24] D. L. Donoho, I. M. Johnstone et al., "Ideal denoising in an orthonormal basis chosen from a library of bases," Comptes rendus de l'Académie des sciences. Série I, Mathématique, vol. 319, no. 12, pp. 1317-1322, 1994.

[25] Q. Tianshu, W. Shuxun, C. Haihua, and D. Yisong, "Adaptive denoising based on wavelet thresholding method," in International Conference on Signal Processing, vol. 1. IEEE, 2002, pp. 120-123.

[26] L. Su, G. Zhao, and R. Zhang, "Translation-invariant wavelet de-noising method with improved thresholding," in IEEE International Symposium on Communications and Information Technology, vol. 1. IEEE, 2005, pp. 619-622.

[27] B. Vidakovic, Statistical Modeling by Wavelets. John Wiley \& Sons, 2009, vol. 503.

[28] H.-Y. Gao, "Wavelet shrinkage denoising using the non-negative garrote," Journal of Computational and Graphical Statistics, vol. 7, no. 4, pp. 469-488, 1998.

[29] C. M. Stein, "Estimation of the mean of a multivariate normal distribution," The Annals of Statistics, pp. 1135-1151, 1981.

[30] S. P. Ghael, A. M. Sayeed, and R. G. Baraniuk, "Improved wavelet denoising via empirical wiener filtering," in Optical Science, Engineering and Instrumentation'97. International Society for Optics and Photonics, 1997, pp. 389-399.

[31] R. Merletti, M. Knaflitz, C. J. De Luca et al., "Electrically evoked myoelectric signals," Critical Reviews in Biomedical Engineering, vol. 19, no. 4, pp. 293-340, 1992.

[32] ARM Holdings plc, "Cortex microcontroller software interface standard," https://github.com/ARM-software/CMSIS_5, 2017, this work was created under the Apache-2.0 license.

[33] G. P. Nason and B. W. Silverman, "The stationary wavelet transform and some statistical applications," in Wavelets and Statistics. Springer, 1995, pp. 281-299.

[34] M. Hakonen, H. Piitulainen, and A. Visala, "Current state of digital signal processing in myoelectric interfaces and related applications," Biomedical Signal Processing and Control, vol. 18, pp. 334-359, 2015.

[35] B. Hudgins, P. Parker, and R. N. Scott, "A new strategy for multifunction myoelectric control," IEEE Transactions on Biomedical Engineering, vol. 40, no. 1, pp. 82-94, 1993.

[36] A. Phinyomark, P. Phukpattaranont, and C. Limsakul, "Feature reduction and selection for EMG signal classification," Expert Systems with Applications, vol. 39, no. 8, pp. 7420-7431, 2012. 
[37] M. Ortiz-Catalan, "Cardinality as a highly descriptive feature in myoelectric pattern recognition for decoding motor volition," Frontiers in Neuroscience, vol. 9, 2015.

[38] S. Micera, J. Carpaneto, and S. Raspopovic, "Control of Hand Prostheses Using Peripheral Information," IEEE Reviews in Biomedical Engineering, vol. 3, pp. 48-68, 2010.

[39] I. Nabney, NETLAB: algorithms for pattern recognition. Springer Science \& Business Media, 2002.

[40] M. Ortiz-Catalan, R. Brånemark, and B. Håkansson, "Biopatrec: A modular research platform for the control of artificial limbs based on pattern recognition algorithms," Source code for biology and medicine, vol. 8, no. 1, p. 11, 2013.

[41] Ortiz-Catalan M., "Biopatrec data repository," https://github.com/ biopatrec/biopatrec/tree/Data_Repository, 2013.

[42] ADS1299-x Low-Noise, 4-, 6-, 8-Channel, 24-Bit, Analog-to-Digital Converter for EEG and Biopotential Measurements, Texas Instruments, July 2012.

[43] E. Mastinu, P. Doguet, Y. Botquin, B. Håkansson, and M. Ortiz-Catalan, "Embedded system for prosthetic control using implanted neuromuscular interfaces accessed via an osseointegrated implant," IEEE Transactions on Biomedical Circuits and Systems, vol. PP, no. 99, pp. 1-11, 2017.

[44] J. Bowman, "Motion artifact generation illustration," 2017, this work was created under CC BY NC-SA.

[45] M. Ortiz-Catalan, F. Rouhani, R. Brånemark, and B. Håkansson, "Offline accuracy: A potentially misleading metric in myoelectric pattern recognition for prosthetic control," in IEEE International Conference Engineering in Medicine and Biology Society, 2015, pp. 1140-1143.

[46] T. A. Kuiken, B. A. Lock, R. D. Lipschutz, L. A. Miller, K. A Stubblefield, and K. B. Englehart, "Targeted Muscle Reinnervation for Real-time Myoelectric Control of Multifunction Artificial Arms," Journal of the American Medical Association, vol. 301, no. 6, pp. 619628, 2009.

[47] S. M. Wurth and L. J. Hargrove, "A real-time comparison between direct control, sequential pattern recognition control and simultaneous pattern recognition control using a fitts' law style assessment procedure,' Journal of Neuroengineering and Rehabilitation, vol. 11, no. 1, p. 91, 2014.

[48] E. A. Clancy, E. L. Morin, and R. Merletti, "Sampling, noise-reduction and amplitude estimation issues in surface electromyography," Journal of Electromyography and Kinesiology, vol. 12, no. 1, pp. 1-16, 2002.

[49] E. Mastinu, J. Ahlberg, E. Lendaro, L. Hermansson, B. Håkansson, and M. Ortiz-Catalan, "An alternative myoelectric pattern recognition approach for the control of hand prostheses: A case study of use in daily life by a dysmelia subject," IEEE Journal of Translational Engineering in Health and Medicine, vol. 6, pp. 1-12, 2018.

[50] L. M. Hermansson, A. G. Fisher, B. Bernspång, and A.-C. Eliasson, "Assessment of capacity for myoelectric control: a new rasch-built measure of prosthetic hand control," Journal of Rehabilitation Medicine, vol. 37, no. 3, pp. 166-71, 2005.

[51] L. Resnik, L. Adams, M. Borgia, J. Delikat, R. Disla, C. Ebner, and L. S. Walters, "Development and evaluation of the activities measure for upper limb amputees," Archives of Physical Medicine and Rehabilitation, vol. 94, no. 3, pp. 488-494, 2013.

[52] C. M. Light, P. H. Chappell, and P. J. Kyberd, "Establishing a standardized clinical assessment tool of pathologic and prosthetic hand function: normative data, reliability, and validity," Archives of Physical Medicine and Rehabilitation, vol. 83, no. 6, pp. 776-783, 2002

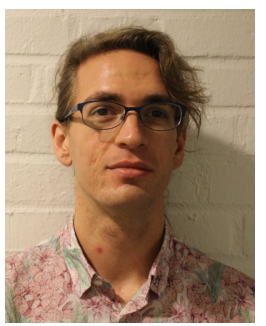

Autumn Naber received a B.Sc. degree in computer engineering from California State University, Sacramento, U.S.A. in 2013 and an M.Sc. in biomedical engineering from Chalmers University of Technology (CTH), Gothenburg, Sweden, in 2017.

She is currently working towards a Ph.D. in biomedical engineering at $\mathrm{CTH}$. Her current research focuses on embedded systems and materials for improving myoelectric control for people with severe neuromuscular disorders.

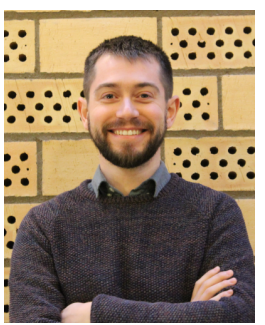

Enzo Mastinu received a M.Sc. degree with top grade in electronic engineering from Universita degli Studi di Cagliari, Italy, in 2014 and a Ph.D. degree in biomedical engineering at Chalmers University of Technology, Sweden, in 2019. During and after the M.Sc., he was HW/FW designer for a spin-off company and a postgraduate research scholarship, respectively.

$\mathrm{He}$ is currently a post-doctoral researcher within the Biomechatronics and Neurorehabilitation Laboratory at Chalmers University of Technology. His research focuses on prosthetic control for upper limb amputees and the research interests cover bioelectric signal acquisition and processing, embedded systems and electronics design, myoelectric pattern recognition, as well as bone-anchored prostheses and sensory feedback via neural stimulation

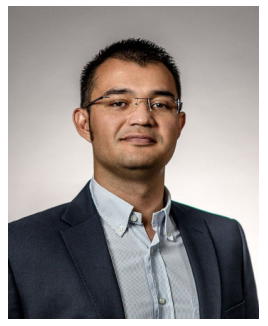

Max Ortiz-Catalan (M'14) was born in Toluca, Mexico in 1982. He received the electronics engineering degree in 2005 from the ITESM Campus Toluca, Mexico, the M.Sc. degree in complex adaptive systems in 2009, and the Ph.D. degree in biomedical engineering in 2014, both from Chalmers University of Technology (CTH), Gothenburg, Sweden.

$\mathrm{He}$ is currently an Associate Professor with CTH where he founded the Biomechatronics and Neurorehabilitation Laboratory (@ChalmersBNL). He also serves as the Research Director with Integrum AB, Sweden. His research interests include bioelectric signals acquisition electronics (analog and digital), signal processing and artificial intelligence algorithms for decoding motor volition and control, neuromuscular interfaces, bone-anchored prostheses and osseointegration, virtual and augmented reality for neuromuscular rehabilitation and the treatment of neuropathic pain.

Dr. Ortiz Catalán has received several honors, notably the European Youth Award in 2014, the Delsys Prize in 2016, the Brian \& Joyce Blatchford Award in 2017, and the Swedish Embedded Award in 2018. 